\title{
Some Direct Decompositions of the Set of Integers
}

\author{
By N. G. de Bruijn
}

1. Introduction. Every positive integer can be uniquely represented in the form

$$
x=\epsilon_{0}+2 \epsilon_{1}+2^{2} \epsilon_{2}+2^{3} \epsilon_{3}+\cdots
$$

with $\epsilon_{i}=0$ or $1, \epsilon_{i}=0$ from a certain point onwards, and the sequence $1,2,2^{2}, \ldots$ is essentially the only one with this property. The situation is entirely different, however, if we require that all integers, positive, negative or zero, be represented. Some of the simplest possibilities are obtained by replacing $1,2,2^{2}, 2^{3}, \cdots$ by 1 , $-2,2^{2},-2^{3}, 2^{4},-2^{5}, \cdots$ or by $1,2,-2^{2}, 2^{3}, 2^{4},-2^{5}, 2^{6}, 2^{7},-2^{8}, \cdots$, but there are very many others. Such decompositions were studied in [1]. In particular, cases were investigated where the sequence has the form $M,-2 N, 2^{2} M,-2^{3} N, 2^{4} M$, $-2^{5} N, \cdots$. It is to this type of decomposition of the set of integers that the present paper is devoted entirely.

It is natural to split the resulting representation of $x$ according to terms with $M$ and terms with $N$, so $x=M s_{1}-2 N s_{2}$, and now we can reformulate the problem in terms of the set $S$ of all possible values that $s_{1}$ and $s_{2}$ can assume.

The set $S$ is defined as the set of all nonnegative integers with the property that, when represented in the scale of 4 , they do not contain 2's or 3's, but only 0 's and 1's. So

$$
\begin{aligned}
S=\{0,1,4,5,16,17,20,21,64,65,68,69,80,81,84,85, \\
\\
256,257,260,261,272,273,276,277,320,321, \cdots\} .
\end{aligned}
$$

Let $X$ denote the set of all integers, positive or negative or zero. The decompositions of $X$ we shall be concerned with are of the type illustrated by the following examples: every $x \in X$ can be represented in exactly one way as

$$
x=s_{1}-2 s_{2} \quad\left(s_{1} \in S, s_{2} \in S\right),
$$

and in exactly one way as

$$
x=7 s_{1}-2 s_{2} \quad\left(s_{1} \in S, s_{2} \in S\right) .
$$

We shall say that a pair $(M, N)$ of nonnegative integers is a good pair if it is true that every $x \in X$ can be represented uniquely as

$$
x=M s_{1}-2 N s_{2} \quad\left(s_{1} \in S, s_{2} \in S\right) .
$$

Saying that $(M, N)$ is good is equivalent to saying that the formal relation

$$
F\left(z^{M}\right) F\left(z^{-2 N}\right)=\sum_{k=-\infty}^{\infty} z^{k}
$$

holds, where

Received January 15, 1964. 


$$
F(z)=(1+z)\left(1+z^{4}\right)\left(1+z^{16}\right)\left(1+z^{64}\right) \cdots .
$$

These good pairs were discussed in [1, Section 4] (there they were called "basic" pairs), as a special case in a more general setting. The following statements were proved there:

(1) If $(M, N)$ is good, then $(N, M)$ is good.

(2) If either $M$ or $N$ is divisible by any number of the form $2^{m}+1(m=$ $0,1,2, \cdots)$, then $(M, N)$ is not good.

(3) For each $k(k=0,1,2, \cdots)$, the pair $\left(1,2^{2 k+1}-1\right)$ is good.

(4) If $(M, N)$ is good, then $M \equiv N(\bmod 6)$.

(5) If $(M, N)$ is good, then $M$ and $N$ have no common divisor.

(6) If $(M, N)$ is good, and $t=N / M$, then $t$ does not belong to any of the following intervals:

$$
1<t<\frac{3}{2}, \quad 3<t<6, \quad 11<t<24, \quad 43<t<96, \quad \cdots .
$$

If we use the fact that either $M=1$ or $M \geqq 7$ (see (2)), the argument can be refined a little, and leads to the conclusion that $t$ does not belong to any of the intervals $2^{2 k+1} / 3 \leqq t \leqq 3 \cdot 2^{2 k-1}(k=0, \pm 1, \pm 2, \cdots)$.

There exists a procedure by which, for each pair $(M, N)$, we can decide in a finite number of steps whether it is good or not. It is a special case of a procedure for a slightly more general situation (see [1]), and can be described as follows. We construct an oriented graph whose vertices are the integers. If $x \in X, x_{1} \in X$, we take an oriented edge from $x$ to $x_{1}$ if and only if one of the following relations holds:

$$
x=4 x_{1}, \quad x=4 x_{1}+M, \quad x=4 x_{1}-2 N, \quad x=4 x_{1}+M-2 N .
$$

Assuming that both $M$ and $N$ are odd (which is obviously necessary for the pair to be good), we notice that to each $x$ there belongs exactly one $x_{1}$.

Removing the loop from 0 to 0 , we have

(7) The pair $(M, N)$ is good if and only if the graph is a tree (whose root is 0 , of course).

We need to investigate only the part of the graph lying in the interval

$$
-M / 3 \leqq x \leqq 2 N / 3 \text {, }
$$

for if $x>2 N / 3$ we have $-M / 3<x_{1}<x$, and if $x<-M / 3$ we have $x<x_{1}<$ $2 N / 3$. So if the part inside (A) is a tree, the whole graph is a tree. We denote the part inside (A) by $\Gamma$.

In [1] we listed all good pairs as far as $1 \leqq M \leqq N \leqq 100$, obtained with the aid of pencil and paper. (This included making a table of the relation between $x$ and $x_{1}$, constructed with four strips of paper that simply had to be shifted in order to switch on the next pair.) The material has now been extended considerably with the assistance of an IBM 1620 computer. The author is indebted to Mrs. E. Simarro who did a large part of the actual programming.

2. Computations and Observations. A pair $(M, N)$ was investigated as follows. We consider the component $\Gamma_{1}$ of $\Gamma$ that contains 0 . This $\Gamma_{1}$ is always a tree, and the question is whether $\Gamma_{1}=\Gamma$. In other words, the question is whether the number of vertices of $\Gamma_{1}$ is equal to the total number of integers in the interval $-M / 3 \leqq$ 
$x \leqq 2 N / 3$. The number of vertices of $\Gamma_{1}$ was obtained by tracing $\Gamma_{1}$ in such a way that each edge is taken just once in one direction, and just once in the other direction. At each point investigated during the tracing, we kept a record of the direct path leading from that point to 0 .

In Table 1 we list all good pairs as far as $M$ and $N$ are at most 1800 . According to (1) we restricted ourselves to $M \leqq N$, and the pairs are arranged with increasing $N$. If either $N$ or $M$ was divisible by 2,3 or 5 (see (2)), or if $N-M$ was not a multiple of 6 (see (4)), the pair was not investigated any further. Also, the pair was dropped if $N / 24 \leqq M \leqq 3 N / 32$, or $N / 6 \leqq M \leqq 3 N / 8$ or $2 N / 3 \leqq M \leqq N$ ( see $(6))$.

Table 1 shows many pairs with $M=1$ (by (3) we know there are infinitely many of them). In a separate investigation we determined all $N \leqq 24889$ such that $(1, N)$ is good. These are shown in Table 2 . The gaps predicted by $(6)$ are clearly visible: between 7 and 31, between 37 and 109, between 679 and 1579, between 2677 and 6493, and between 10879 and 24649. For all $N \leqq 24889$ (restricted to $N \equiv 1(\bmod 6), N \not \equiv 0(\bmod 5))$ we determined the number of vertices of the subtree $\Gamma_{1}$, but these numbers are not listed in this paper. Inside the gaps as indicated by (7), this number of vertices is constant, and in fact, the tree undergoes deformations only if $N$ runs through such intervals, and the topological structure is unaltered. $\Gamma_{1}$ has 2 vertices if $N=13$ or 19,4 vertices if $43 \leqq N \leqq 91,8$ vertices if $181 \leqq N \leqq 379,16$ vertices if $691 \leqq N \leqq 1531,32$ vertices if $2731 \leqq N \leqq 6139$, 64 vertices if $10927 \leqq N \leqq 24571$. This is easily proved; we do not need a computer for this.

There are also other gaps in the list, again corresponding to trees with constant shape, but slightly more complicated than in the cases just mentioned. For example, there are 40 vertices if $1777 \leqq N \leqq 1879,48$ vertices if $2221 \leqq N \leqq 2359,80$ vertices if $7111 \leqq N \leqq 7559,96$ vertices if $8881 \leqq N \leqq 9451$.

Another look at Table 1 shows that there are many good pairs where $N=$ $2 M+1$ or $N=2 M-1$. These were investigated separately up to $M=8171$, and listed in Table 3. Also here, we notice a number of gaps, which can easily be discussed theoretically: if $4^{k}<M<6 \cdot 4^{k-1}, N=2 M \pm 1$, then the subtree $\Gamma_{1}$ has exactly $2^{k}$ vertices.

Some good pairs look particularly pretty. For example:

$$
(7,13),\left(7,13^{2}\right) ; \quad\left(7^{2}, 11^{2}\right),\left(1,11^{2}\right),\left(1,23^{2}\right) ; \quad(1,7),\left(1,7^{2}\right),\left(1,7^{4}\right) .
$$

A more careful inspection of the available material led to the discovery of some infinite sequences of good pairs. The pairs

$$
(11,23),(11,89),(11,353),(11,1409) \text {, }
$$

all of the form $\left(11,22 \cdot 4^{k}+1\right)$, can be found in Table 1, and the computer proved that the next three items, viz. $(11,5633),(11,22529),(11,90113)$ are also good. A general proof is presented below (Theorem 1).

Another remarkable sequence can be conjectured from Table 2, viz. $\left(1,10 \cdot 4^{k}-3\right)$, of which Table 2 shows the first six cases, viz.

$$
(1,7),(1,37),(1,157),(1,637),(1,2557),(1,10237) \text {. }
$$

See Theorem 2 for a general proof. 
TABLE 1

Good pairs with $1 \leqq M \leqq N \leqq 1800$

\begin{tabular}{|c|c|c|c|c|c|c|c|c|c|}
\hline$M$ & $N$ & $M$ & $N$ & $M$ & $N$ & $M$ & $N$ & $M$ & $N$ \\
\hline 1 & 1 & 7 & 277 & 311 & 539 & 373 & 781 & 521 & 1043 \\
\hline 1 & 7 & 139 & 277 & 293 & 563 & 511 & 781 & 683 & 1043 \\
\hline 7 & 13 & 47 & 287 & 373 & 571 & 397 & 787 & 131 & 1049 \\
\hline 11 & 23 & 143 & 287 & 91 & 583 & 397 & 793 & 667 & 1051 \\
\hline 1 & 31 & 151 & 301 & 343 & 583 & 311 & 803 & 31 & 1057 \\
\hline 13 & 31 & 31 & 307 & 23 & 587 & 491 & 809 & 131 & 1061 \\
\hline 19 & 31 & 161 & 317 & 59 & 587 & 511 & 817 & 7 & 1063 \\
\hline 1 & 37 & 211 & 319 & 367 & 589 & 499 & 823 & 133 & 1063 \\
\hline 7 & 43 & 163 & 331 & 293 & 593 & 349 & 829 & 131 & 1067 \\
\hline 31 & 49 & 13 & 337 & 23 & 599 & 7 & 841 & 103 & 1069 \\
\hline 31 & 61 & 127 & 337 & 347 & 599 & 7 & 853 & 11 & 1073 \\
\hline 31 & 67 & 43 & 343 & 371 & 599 & 83 & 857 & 113 & 1073 \\
\hline 47 & 71 & 199 & 343 & 19 & 601 & 367 & 859 & 29 & 1079 \\
\hline 7 & 73 & 211 & 343 & 61 & 607 & 547 & 877 & 539 & 1079 \\
\hline 31 & 73 & 11 & 347 & 317 & 611 & 413 & 881 & 157 & 1081 \\
\hline 41 & 77 & 173 & 347 & 287 & 617 & 7 & 883 & 679 & 1087 \\
\hline 47 & 77 & 11 & 353 & 19 & 619 & 383 & 887 & 167 & 1091 \\
\hline 41 & 83 & 179 & 359 & 401 & 623 & 437 & 887 & 109 & 1093 \\
\hline 11 & 89 & 157 & 367 & 1 & 631 & 467 & 887 & 529 & 1093 \\
\hline 49 & 97 & 229 & 367 & 1 & 637 & 139 & 889 & 547 & 1093 \\
\hline 41 & 101 & 181 & 379 & 61 & 637 & 541 & 889 & 137 & 1097 \\
\hline 49 & 103 & 61 & 397 & 419 & 641 & 419 & 893 & 667 & 1099 \\
\hline 1 & 109 & 157 & 397 & 61 & 643 & 7 & 907 & 443 & 1103 \\
\hline 1 & 121 & 53 & 401 & 299 & 647 & 109 & 919 & 107 & 1109 \\
\hline 49 & 121 & 203 & 401 & 1 & 661 & 389 & 923 & 137 & 1109 \\
\hline 79 & 121 & 1 & 403 & 97 & 661 & 31 & 931 & 139 & 1111 \\
\hline 1 & 127 & 203 & 407 & 311 & 671 & 541 & 931 & 43 & 1117 \\
\hline 79 & 127 & 193 & 409 & 413 & 671 & 7 & 937 & 7 & 1123 \\
\hline 1 & 133 & 217 & 409 & 419 & 671 & 91 & 937 & 589 & 1123 \\
\hline 89 & 143 & 11 & 419 & 349 & 673 & 7 & 943 & 691 & 1123 \\
\hline 1 & 151 & 1 & 421 & 421 & 673 & 97 & 943 & 167 & 1127 \\
\hline 79 & 151 & 199 & 427 & 293 & 677 & 553 & 943 & 701 & 1127 \\
\hline 1 & 157 & 211 & 427 & 1 & 679 & 491 & 947 & 457 & 1129 \\
\hline 103 & 157 & 217 & 433 & 107 & 683 & 31 & 949 & 719 & 1133 \\
\hline 89 & 167 & 181 & 451 & 23 & 701 & 511 & 961 & 37 & 1141 \\
\hline 7 & 169 & 229 & 451 & 371 & 701 & 367 & 967 & 133 & 1147 \\
\hline 7 & 193 & 67 & 457 & 83 & 713 & 457 & 967 & 151 & 1153 \\
\hline 91 & 193 & 287 & 461 & 89 & 719 & 491 & 971 & 463 & 1153 \\
\hline 121 & 193 & 47 & 467 & 107 & 719 & 517 & 973 & 571 & 1159 \\
\hline 31 & 199 & 19 & 469 & 73 & 727 & 31 & 979 & 37 & 1171 \\
\hline 121 & 199 & 73 & 469 & 97 & 733 & 373 & 979 & 463 & 1171 \\
\hline 89 & 209 & 251 & 473 & 307 & 733 & 491 & 983 & 469 & 1171 \\
\hline 101 & 209 & 59 & 479 & 451 & 733 & 157 & 997 & 589 & 1177 \\
\hline 131 & 209 & 193 & 487 & 121 & 739 & 167 & 1007 & 733 & 1177 \\
\hline 7 & 211 & 263 & 497 & 23 & 749 & 499 & 1009 & 149 & 1187 \\
\hline 103 & 217 & 199 & 499 & 403 & 757 & 161 & 1019 & 587 & 1187 \\
\hline 109 & 217 & 1 & 511 & 19 & 763 & 401 & 1019 & 161 & 1199 \\
\hline 7 & 223 & 61 & 511 & 127 & 763 & 37 & 1021 & 599 & 1199 \\
\hline 31 & 229 & 67 & 511 & 463 & 763 & 511 & 1021 & 751 & 1201 \\
\hline 137 & 233 & 247 & 511 & 77 & 767 & 511 & 1027 & 611 & 1211 \\
\hline 127 & 253 & 209 & 521 & 451 & 769 & 137 & 1031 & 713 & 1211 \\
\hline 163 & 253 & 1 & 529 & 503 & 773 & 43 & 1033 & 499 & 1213 \\
\hline 29 & 269 & 83 & 539 & 337 & 781 & 667 & 1033 & 647 & 1217 \\
\hline
\end{tabular}


TABLE 1-Continued

\begin{tabular}{rl|rc|rc|rr|rr}
\hline \multicolumn{1}{r}{$M$} & $N$ & \multicolumn{1}{|c|}{$M$} & $N$ & $M$ & $N$ & $M$ & $N$ & \multicolumn{1}{|c}{$M$} & $N$ \\
\hline 49 & 1219 & 211 & 1369 & 149 & 1493 & 997 & 1603 & 733 & 1693 \\
571 & 1219 & 43 & 1381 & 719 & 1499 & 803 & 1607 & 647 & 1697 \\
589 & 1219 & 683 & 1391 & 749 & 1499 & 199 & 1609 & 1109 & 1697 \\
149 & 1247 & 139 & 1393 & 59 & 1511 & 767 & 1613 & 727 & 1699 \\
479 & 1247 & 11 & 1409 & 203 & 1523 & 1 & 1621 & 53 & 1703 \\
479 & 1253 & 707 & 1409 & 983 & 1529 & 193 & 1621 & 173 & 1703 \\
13 & 1267 & 217 & 1417 & 721 & 1537 & 763 & 1621 & 1061 & 1703 \\
149 & 1271 & 47 & 1421 & 907 & 1537 & 869 & 1631 & 11 & 1709 \\
161 & 1283 & 749 & 1427 & 893 & 1541 & 1 & 1633 & 851 & 1709 \\
31 & 1291 & 547 & 1429 & 181 & 1543 & 203 & 1637 & 1061 & 1721 \\
41 & 1307 & 47 & 1433 & 917 & 1559 & 857 & 1637 & 11 & 1733 \\
623 & 1307 & 167 & 1433 & 181 & 1561 & 667 & 1639 & 271 & 1741 \\
167 & 1319 & 851 & 1433 & 817 & 1561 & 809 & 1643 & 283 & 1753 \\
647 & 1319 & 581 & 1451 & 193 & 1567 & 661 & 1651 & 11 & 1757 \\
821 & 1319 & 737 & 1451 & 197 & 1571 & 791 & 1661 & 167 & 1757 \\
823 & 1321 & 583 & 1453 & 971 & 1571 & 13 & 1663 & 911 & 1757 \\
133 & 1327 & 197 & 1457 & 743 & 1577 & 13 & 1669 & 73 & 1759 \\
691 & 1333 & 173 & 1463 & 791 & 1577 & 259 & 1669 & 211 & 1771 \\
137 & 1349 & 587 & 1463 & 1 & 1579 & 251 & 1679 & 937 & 1771 \\
199 & 1351 & 589 & 1471 & 803 & 1583 & 677 & 1679 & 1123 & 1771 \\
517 & 1351 & 199 & 1477 & 631 & 1591 & 223 & 1687 & 1091 & 1787 \\
43 & 1357 & 11 & 1481 & 991 & 1597 & 53 & 1691 & 13 & 1789 \\
683 & 1367 & 737 & 1481 & 817 & 1603 & 899 & 1691 & 899 & 1793 \\
163 & 1369 & & & & & & & & \\
\hline
\end{tabular}

TABLE 2

Values of $N$ (with $1 \leqq N \leqq 24889$ ) such that $(1, N)$ is a good pair

\begin{tabular}{r|r|r|r|l|l|l|l|l|l|r|r}
\hline 1 & 151 & 661 & 2041 & 2173 & 6661 & 7591 & 8089 & 8311 & 9487 & 9991 & 10669 \\
7 & 157 & 679 & 2047 & 2401 & 6703 & 7717 & 8101 & 8317 & 9493 & 10087 & 10741 \\
31 & 403 & 1579 & 2053 & 2527 & 6733 & 7729 & 8173 & 8497 & 9601 & 10111 & 10837 \\
37 & 421 & 1621 & 2071 & 2557 & 6871 & 7747 & 8191 & 8569 & 9631 & 10159 & 10879 \\
109 & 511 & 1633 & 2077 & 2677 & 6967 & 7753 & 8197 & 8623 & 9757 & 10237 & 24649 \\
121 & 529 & 1969 & 2143 & 6493 & 6973 & 7819 & 8221 & 8701 & 9937 & 10261 & 24751 \\
127 & 631 & 1981 & 2149 & 6559 & 6979 & 7861 & 8257 & 9457 & 9961 & 10663 & 24781 \\
133 & 637 & 2017 & 2167 & 6643 & 7009 & & & & & & \\
\hline
\end{tabular}

TABLE 3

Good pairs $(M, N)$ with $N=2 M \pm 1 ; 1 \leqq M \leqq 8171$

\begin{tabular}{|c|c|c|c|c|c|c|c|c|c|}
\hline$M$ & $N$ & $M$ & $N$ & $M$ & $N$ & $M$ & $N$ & $M$ & $N$ \\
\hline 1 & 1 & 143 & 287 & 521 & 1043 & 989 & 1979 & 2723 & 5447 \\
\hline 7 & 13 & 151 & 301 & 539 & 1079 & 2041 & 4081 & 2761 & 5521 \\
\hline 11 & 23 & 173 & 347 & 547 & 1093 & 2047 & 4093 & 2999 & 5999 \\
\hline 31 & 61 & 179 & 359 & 589 & 1177 & 2107 & 4213 & 3661 & 7321 \\
\hline 41 & 83 & 203 & 407 & 599 & 1199 & 2153 & 4307 & 3721 & 7441 \\
\hline 49 & 97 & 217 & 433 & 683 & 1367 & 2191 & 4381 & 3739 & 7477 \\
\hline 109 & 217 & 397 & 793 & 749 & 1499 & 2219 & 4439 & 6199 & 12397 \\
\hline 127 & 253 & 491 & 983 & 803 & 1607 & 2531 & 5063 & 6923 & 13847 \\
\hline 139 & 277 & 511 & 1021 & 929 & 1859 & 2693 & 5387 & 7949 & 15899 \\
\hline
\end{tabular}


In Table 3 we can discover the sequence

$$
(1,1),(7,13),(31,61),(127,253),(511,1021),(2047,4093) \text {, }
$$

i.e., the first six items of the sequence $\left(2^{2 k-1}-1,2^{2 k}-3\right)$. See Theorem 3 for a general proof.

The case $(11,90113)$ was the "largest" one presented to the computer during this investigation. In that case we have a very large tree, filling the whole interval $[-30037,+7]$ (see (A)), and it took the computer 14 minutes to make sure that the tree has 30045 nodes indeed. The "height" of this tree is comparatively small: its highest point -21838 has level 32 , i.e., iteration of the mapping $x \rightarrow x_{1}$, starting with $x=-21838$, produces 0 in 32 steps: $-21838 \rightarrow-5454 \rightarrow-1358 \rightarrow-334 \rightarrow$ $-78 \rightarrow-14 \rightarrow+2 \rightarrow+6 \rightarrow+7 \rightarrow-22521 \rightarrow-28153 \rightarrow-29561 \rightarrow-29913 \rightarrow$ $-30001 \rightarrow-30023 \rightarrow-30034 \rightarrow-7503 \rightarrow-24404 \rightarrow-6101 \rightarrow-24048 \rightarrow$ $-6012 \rightarrow-1503 \rightarrow-22904 \rightarrow-5726 \rightarrow-1426 \rightarrow-351 \rightarrow-22616 \rightarrow-5654 \rightarrow$ $-1408 \rightarrow-352 \rightarrow-88 \rightarrow-22 \rightarrow 0$.

3. Three Theorems. As a preparation for Theorems 1, 2, 3, we first prove two lemmas.

Lemma 1. If a pair $(M, N)$ is not a good pair then there exists a positive integer $n$, and there exist numbers $\epsilon_{1}, \cdots, \epsilon_{n}, \delta_{1}, \cdots, \delta_{n}$, all either 0 or 1 , but not all 0 , such that

$$
\sum_{i=1}^{n}\left(M \epsilon_{i}-2 N \delta_{i}\right) 4^{i-1} \equiv 0\left(\bmod 4^{n}-1\right) .
$$

Proof (cf. [1], Theorem 7). It follows from (7) that if $\Gamma$ is not a tree, then the interval (A) has to contain a closed cycle of $\Gamma$, viz. $x=x_{0} \rightarrow x_{1} \rightarrow x_{2} \rightarrow \cdots \rightarrow$ $x_{n}=x_{0}$ and $x_{i}=4 x_{i+1}+M \epsilon_{i}-2 N \delta_{i}$, with suitable values of $\epsilon_{i}$ and $\delta_{i}$. It follows that

$$
\sum_{i=1}^{n}\left(M \epsilon_{i}-2 N \delta_{i}\right) 4^{i-1}=-\left(4^{n}-1\right) x_{0} .
$$

Since the loop $0 \rightarrow 0$ was removed from our graph, we have $x_{0} \neq 0$; this excludes $\epsilon_{1}=\cdots=\epsilon_{n}=\delta_{1}=\cdots=\delta_{n}=0$.

Lemma 2. Let $n, k, \epsilon_{1}, \cdots, \epsilon_{n}$ be integers ( $\left.n \geqq 1\right)$. If $p$ is an integer, we define $\{p\}$ as the number defined by $\{p\} \equiv p(\bmod n), 0<\{p\} \leqq n$. Then we have

$$
4^{k} \sum_{i=1}^{n} \epsilon_{i} 4^{i-1} \equiv \sum_{i=1}^{n} \epsilon_{\{i-k\}} 4^{i-1}\left(\bmod 4^{n}-1\right) .
$$

Proof. We have $4^{k+i-1} \equiv 4^{\{k+i\}-1}$; moreover

$$
\sum_{i=1}^{n} \epsilon_{\{i\}} 4^{\{k+i\}-1}=\sum_{i=k+1}^{n+k} \epsilon_{\{i-k\}} 4^{\{i\}-1}=\sum_{i=1}^{n} \epsilon_{\{i-k\}} 4^{\{i\}-1} .
$$

Lemma 3. Let $n, \zeta_{1}, \cdots, \zeta_{n}$ be integers, and assume that

$$
\sum_{i=1}^{n} \zeta_{i} 4^{i-1} \equiv 0\left(\bmod 4^{n}-1\right) .
$$

Then there exist integers $t_{0}, t_{1}, \cdots, t_{n}=t_{0}$ such that 


$$
\begin{aligned}
\frac{1}{3} \min _{i} \zeta_{i} & \leqq t_{j} \leqq \frac{1}{3} \max _{i} \zeta_{i} \quad(j=0, \cdots, n) \\
t_{j+1} & =4 t_{j}-\zeta_{\{-j\}} \quad(j=0, \cdots, n-1) .
\end{aligned}
$$

Proof. For each $j$ we put

$$
w_{j}=\sum_{i=1}^{n} \zeta_{\{i-j\}} 4^{i-1}
$$

and we easily obtain $w_{j+1}=4 w_{j}-\left(4^{n}-1\right) \zeta_{\{-j\}}$. Furthermore we have

$$
w_{j} \leqq\left(\max _{i} \zeta_{i}\right)\left(1+4+\cdots+4^{n-1}\right)=\frac{1}{3}\left(\max _{i} \zeta_{i}\right)\left(4^{n}-1\right),
$$

and a similar lower estimate. So taking $t_{j}=w_{j} /\left(4^{j}-1\right)$, we have proved our lemma.

Theorem 1. If $k=0,1,2, \cdots$, the pair $\left(22 \cdot 4^{k}+1,11\right)$ is good.

Proof. Assume that this is false for some $k$. Apply Lemma 1 , with $M=22 \cdot 4^{k}+1$, $N=11$; this produces $\epsilon_{1}, \cdots, \epsilon_{n}, \delta_{1}, \cdots, \delta_{n}$, all 0 or 1 , and not all 0 . We split $M$ into the two parts 1 and $22 \cdot 4^{k}$; to the second part we apply Lemma 2 . Thus we obtain

$$
\sum_{i=1}^{n}\left(\epsilon_{i}+22\left(\epsilon_{\{i-k\}}-\delta_{i}\right)\right) 4^{i-1} \equiv 0\left(\bmod 4^{n}-1\right) .
$$

To this sum we apply Lemma 3, with $\zeta_{i}=\epsilon_{i}+22\left(\epsilon_{\{i-k\}}-\delta_{i}\right)$. That produces the cycle $t_{0}, t_{1}, \cdots, t_{n}=t_{0}$. We have $\zeta_{i} \in Z=\{0,22,-22,1,23,-21\}$, so by Lemma 3 the $t$ 's are restricted by $-7 \leqq t_{j} \leqq 7, t_{j+1}-4 t_{j} \in Z$. We can now make a list of all possibilities for the vector $\left(t_{j}, t_{j+1}, \zeta_{\{-j\}}\right)$. They are

$$
\begin{aligned}
& (-7,-7,-21),(-7,-6,-22),(-6,-3,-21),(-6,-2,-22), \\
& (-5,1,-21),(-5,2,-22),(-4,5,-21),(-4,6,-22),(-1,-5,1), \\
& (-1,-4,0),(0,-1,1),(0,0,0),(1,3,1),(1,4,0),(2,7,1),(4,-7,23), \\
& (4,-6,22),(5,-3,23),(5,-2,22),(6,1,23),(6,2,22),(7,5,23), \\
& (7,6,22) .
\end{aligned}
$$

The possible transitions from a $t_{j}$ to the next one, $t_{j+1}$, can be visualized by drawing a graph with the nodes $-7, \cdots,+7$, whose oriented edges represent the transitions $t_{j} \rightarrow t_{j+1}$ that can be obtained from the above vectors: $(-7,-7),(-7,-6)$, $(-6,-3),(-6,-2),(-5,1),(-5,2),(-4,-5),(-4,-6),(-1,-5)$, $(-1,-4),(0,-1),(0,0),(1,3),(1,4),(2,7),(4,-7),(4,-6),(5,-3)$, $(5,-2),(6,1),(6,2),(7,5),(7,6)$. The cycle $t_{0} \rightarrow t_{1} \rightarrow \cdots \rightarrow t_{n}=t_{0}$ represents some closed circuit in this oriented graph. It is easy to see that there can be only the following three closed circuits: (a) $\cdots \rightarrow 0 \rightarrow 0 \rightarrow 0 \rightarrow \cdots$, (b) $\cdots \rightarrow$ $-7 \rightarrow-7 \rightarrow-7 \rightarrow \cdots,(\mathrm{c}) \cdots \rightarrow 2 \rightarrow 7 \rightarrow 6 \rightarrow 2 \rightarrow 7 \rightarrow 6 \rightarrow \cdots$; in case (c) it is obvious that $n$ is a multiple of 3 .

In case (a) we have $t_{j}=t_{j+1}=0$ for all $j$, whence $\zeta_{i}=0$ for all $i$. By $\zeta_{i}=\epsilon_{i}+$ $22\left(\epsilon_{\{i-k\}}-\delta_{i}\right)$ we deduce that $\epsilon_{1}=\cdots=\epsilon_{n}=\delta_{1}=\cdots=\delta_{n}=0$. This contradicts our assumption, so case (a) is impossible.

In case (b) we have $t_{j}=t_{j+1}=-7$ for all $j$, whence $\zeta_{i}=-21$ for all $j$, so 
$\epsilon_{i}=1, \epsilon_{\{i-k\}}=0, \delta_{i}=1$ for all $i$. This is impossible, for if $\epsilon_{i}=1$ for all $i$ then $\epsilon_{\{i-k\}}=1$ for all $i$. This excludes case (b).

We finally consider case (c). The transitions $2 \rightarrow 7,7 \rightarrow 6,6 \rightarrow 2$ correspond to the vectors $(2,7,1),(7,6,22),(6,2,22)$, respectively. If $\zeta_{i}=1$ we have $\epsilon_{i}=1$, $\epsilon_{\{i-k\}}-\delta_{i}=0$, if $\zeta_{i}=22$ we have $\epsilon_{i}=0, \epsilon_{\{i-k\}}-\delta_{i}=1$. Therefore, the average value of $\epsilon_{i}$ is $\frac{1}{3}$, and the average of $\epsilon_{\{i-k\}}-\delta_{i}$ is $\frac{2}{3}$. It follows that the average of $\delta_{i}$ is $-\frac{1}{3}$, which is impossible. This excludes (c), which was the one last possibility, and our proof is complete.

Theorem 2. If $k=0,1,2, \cdots$, the pair $\left(10 \cdot 4^{k}-3,1\right)$ is good.

Proof. The proof is similar to the one of Theorem 1. Assume that, for some $k$, $\left(10 \cdot 4^{k}-3,1\right)$ is not good. Apply Lemma 1 , with $M=10 \cdot 4^{k}-3, N=1$; this again produces $\epsilon_{1}, \cdots, \epsilon_{n}, \delta_{1}, \cdots, \delta_{n}$, all 0 or 1 , and not all 0 . We obtain, using Lemma 2,

$$
\sum_{i=1}^{n}\left(-3 \epsilon_{i}+10 \epsilon_{\{i-k\}}-2 \delta_{i}\right) 4^{i-1} \equiv 0\left(\bmod 4^{n}-1\right) .
$$

We apply Lemma 3 with $\zeta_{i}=-3 \epsilon_{i}+10 \epsilon_{\{i-k\}}-2 \delta_{i}$. As in the proof of Theorem 1 , we obtain $Z=\{0,10,8,-2,-3,7,5,-5\}$; the restriction on the elements of the cycle $t_{0} \rightarrow t_{1} \rightarrow \cdots \rightarrow t_{n}=t_{0}$ is $-1 \leqq t_{j} \leqq 3$, and the possible vectors $\left(t_{j}, t_{j+1}, \zeta_{\{-j\}}\right)$ are

$$
\begin{aligned}
& (-1,-1,-3),(-1,1,-5),(0,0,0),(0,2,-2),(0,3,-3),(1,-1,5), \\
& (2,0,8),(2,1,7),(2,3,5),(3,2,10) .
\end{aligned}
$$

Drawing the transition graph with nodes $-1,0,1,2,3$ and oriented edges $(-1,-1)$, $(-1,1),(0,0),(0,2),(0,3),(1,-1),(2,0),(2,1),(2,3),(3,2)$, we notice that the $t$-cycle has to lie either in the subgraph determined by the nodes $0,2,3$ or in the subgraph determined by $1,-1$, because there is no way leading from the second group to the first one.

First we assume that our cycle lies in the first group. For the transitions inside this group we evaluate $\tau_{i}=\epsilon_{\{i-k\}}-\epsilon_{i}$. Each value of $\zeta_{i} \in Z$ uniquely determines $\epsilon_{i}, \epsilon_{\{i-k\}}$ and $\delta_{i}$, and we obtain that $\tau_{i}=0,1,1,0,-1,0,0,-1$ if $\zeta_{i}=0,10,8$, $-2,-3,7,5,-5$, respectively. It follows that the transitions $0 \rightarrow 0,0 \rightarrow 2,2 \rightarrow 0$, $0 \rightarrow 3,2 \rightarrow 3,3 \rightarrow 2$ correspond to $\tau_{i}=0,0,1,-1,0,1$, respectively. The average of all $\tau_{i}$ has to be 0 (since $\epsilon_{i}$ and $\epsilon_{\{i-k\}}$ have the same average). It is obvious from the graph that if our cycle contains any transition other than $0 \rightarrow 0$, then it has to contain $2 \rightarrow 0$, where $\tau_{i}=1$. This spoils the average: the value -1 on $0 \rightarrow 3$ cannot compensate for this, for $0 \rightarrow 3$ is necessarily followed by $3 \rightarrow 2$, and the joint contribution of $0 \rightarrow 3$ and $3 \rightarrow 2$ to the sum of the $\tau$ 's is 0 . Thus there only remains the cycle $\cdots \rightarrow 0 \rightarrow 0 \rightarrow 0 \rightarrow \cdots$. But this cycle leads to $\epsilon_{1}=\cdots=\epsilon_{n}=$ $\delta_{1}=\cdots=\delta_{n}=0$, which was excluded.

We next assume that our cycle lies in the second group, where we have the transitions $-1 \rightarrow-1,-1 \rightarrow 1,1 \rightarrow-1$. These correspond to the values $\tau_{i}=$ $-1,-1,0$, respectively. So it follows that in any cycle all $\tau_{i}$ are $\leqq 0$, some are $<0$, and the average cannot be 0 . This completes the proof.

Theorem 3. If $k=1,2,3, \cdots$, the pair $\left(2^{2 k}-3,2^{2 k-1}-1\right)$ is good.

Proof. We follow the same pattern as in the proofs of the previous theorems. 
The graph is much simpler in this case, but the reasoning has to be more delicate since the averaging argument fails.

Assuming that for some $k$ the pair is not good, we obtain, using Lemmas 1 and 2

$$
\sum_{i=1}^{n}\left(-3 \delta_{i}+\delta_{\{i-k\}}+2 \epsilon_{i}-\epsilon_{\{i-k\}}\right) 4^{i-1} \equiv 0\left(\bmod 4^{n}-1\right) .
$$

We put

$$
\epsilon_{i}-\delta_{i}=\sigma_{i}, \quad \zeta_{i}=3 \sigma_{i}-\sigma_{\{i-k\}}-\epsilon_{i},
$$

and we apply Lemma 3 , producing a cycle $t_{0}, t_{1}, \cdots, t_{n}=t_{0}$. We have $4 t_{j}-$ $t_{j+1}=\zeta_{\{-j\}} \in Z$, and $Z=\{-4,-3,-2,-1,0,1,2,3\}$, whence $-1 \leqq t_{j} \leqq 1$. The possible vectors $\left(t_{j}, t_{j+1}, \zeta_{\{-j\}}\right)$ are

$$
(1,1,3),(0,1,-1),(0,0,0),(0,-1,1),(-1,0,-4),(-1,-1,-3) \text {. }
$$

The only vector whose first entry equals 1 is $(1,1,3)$. So if $t_{j}=1$ for one $j$, then $t_{j}=1$ for all $j$, and $\zeta_{\{-j\}}=3$ for all $j$. It follows that $\sigma_{i}=1$ for all $i$, so $\sigma_{\{i-k\}}=$ 1 for all $i$, whence $\epsilon_{i}=3-1-3=-1$ for all $i$. This is impossible. Henceforth we shall assume that $t_{j} \neq 1$ for all $j$.

First we take the case that all $t_{j}=0$ for all $j$, so $\zeta_{i}=0$ for all $i$. Hence $3 \sigma_{i}=$ $\sigma_{\{i-k\}}+\epsilon_{i}$ for all $i$; since $\sigma_{j} \in\{-1,0,1\}, \epsilon_{j} \in\{0,1\}$ for all $j$, we deduce that $\sigma_{i}=$ \pm 1 is impossible. So $\sigma_{i}=0$ for all $i$, therefore $\sigma_{\{i-k\}}=0$ for all $i$, hence $\epsilon_{i}=0$ for all $i$. Therefore $\delta_{i}=\epsilon_{i}+\sigma_{i}=0$ for all $i$, and we have reached $\epsilon_{1}=\cdots=\epsilon_{n}=$ $\delta_{1}=\cdots=\delta_{n}=0$, which was excluded beforehand.

Next we take the case that $t_{j}=-1$ for all $j$, so $\zeta_{i}=-3$ for all $i$. From the definition of $\zeta_{i}$ it follows that $\sigma_{i}=-1$ for all $i$. Therefore, $\sigma_{\{i-k\}}=-1$ for all $i$, and we arrive at $-3=-3+1-\epsilon_{i}$, so $\epsilon_{i}=1$ for all $i$. Finally, $\delta_{i}=\epsilon_{i}-\sigma_{i}=2$, but this is impossible since $\delta_{i}$ can be only 0 or 1 .

It remains to investigate the case that some $t_{j}$ are 0 and some are -1 . Then there is a number $m$ with $t_{m}=-1, t_{m+1}=0$. Hence $\zeta_{\{-m\}}=-4$, and it follows that $\sigma_{\{-m\}}=-1$, hence $\epsilon_{\{-m\}}=0, \delta_{\{-m\}}=1$, hence $\sigma_{\{-m-k\}}=-\zeta_{\{-m\}}+3 \sigma_{\{-m\}}-$ $\epsilon_{\{-m\}}=1$. Thus we proved the existence of an index $j$ with $\sigma_{j}=1$.

From the fact that the $t_{i}$ 's only take the values 0 and -1 we deduce:

$$
\text { If } \sigma_{\{-j\}}=1 \text { then } t_{j}=0, t_{j+1}=-1, \sigma_{\{-j-k\}}=1 \text {. }
$$

For, $\sigma_{\{-j\}}=1$ excludes the values $\zeta_{\{-j\}}=-1,0,-4,-3$, and this leaves for $\left(t_{j}, t_{j+1}, \zeta_{\{-j\}}\right)$ only the possibility $(0,-1,1)$. So $\zeta_{\{-j\}}=1$; combined with $\sigma_{\{-j\}}=1$ this leads to $\sigma_{\{-j-k\}}+\epsilon_{\{-j\}}=2$, whence $\sigma_{\{-j-k\}}=1, \epsilon_{\{-j\}}=1$.

We also need:

$$
\text { If } t_{l}=-1, t_{l+1}=0 \text { then } \sigma_{\{-l-k\}}=1 .
$$

For, $\zeta_{\{-l\}}=4 t_{l}-t_{l+1}=-4$, whence $\sigma_{\{-l\}}=-1, \sigma_{\{-l-k\}}+\epsilon_{\{-l\}}=1$. As $\epsilon_{i}=$ $\sigma_{i}+\delta_{i}$, we have $\epsilon_{\{-l\}} \leqq \sigma_{\{-l\}}+1=0$, whence $\epsilon_{\{-l\}}=0, \sigma_{\{-l-k\}}=1$.

We now finish the proof of the theorem. We know that at least once $\sigma_{\{-m\}}=-1$, and that at least once $t_{j}=0$.

Let $h$ be the smallest positive number such that $m$ exists with

$$
\sigma_{\{-m\}}=1, \quad t_{m+h}=0 .
$$


By (B) we have $t_{m+1}=-1$, whence $h>1$. Therefore $t_{m+h-1}=-1$, because of the minimality of $h$.

From (B) we infer $\sigma_{\{-m-k\}}=1$, and if we again apply (B), now with $j=m+k$, we obtain $t_{m+k}=0, t_{m+k+1}=-1$.

Applying (C) with $l=m+h-1$ we deduce $\sigma_{\{-m-h-k+1\}}=1$. Next, applying (B) with $j=m+h+k-1$, we find $t_{m+h+k-1}=0, t_{m+h+k}=1$. Thus we have obtained

$$
\sigma_{\{-m-k\}}=1, \quad t_{m+k+h-1}=0, \quad h-1>0,
$$

and this contradicts the minimality property of $h$. The proof is now complete.

Technological University

Eindhoven, Netherlands

1. N. G. De Bruijn, "On bases for the set of integers," Publ. Math. Debrecen, v. 1, 1950 p. $232-242$. 